\title{
Intraprostatic fossa foley balloon tamponade: a novel technique for controlling of life-threatening bleeding during simple prostatectomy
}

\begin{abstract}
Objective: To evaluated the efficacy, postoperative morbidity and technical complications of novel technique for controlling of bleeding during simple prostatectomy.

Materials and methods: We retrospectively reviewed the 11 patients whom underwent balloon tamponade due to massive life-threatening bleeding from prostatic fossa during open prostatectomy in our center since January 2007 till February 2016

Results: Overall 11 patients were studied. The mean patient age was 74.81 years (range: 64-92). The procedures were performed via a suprapubic transvesical approach in 9 and retropubic in 2 patients. After this simple technique life-threatening bleeding was stopped completely. Fortunately, we didn't find any complication attributable to our technique. There was no bladder neck stricture, fistula formation or rectal injury even in those patients who underwent retropubic procedure. Intra operative transfusion was done in all patients.

Conclusion: Balloon tamponade is a simple, quick and lifesaving new technique which is used when persistent and significant bleeding from the prostatic fossa continues after simple prostatectomy.
\end{abstract}

Keywords: foley balloon tamponade, open prostatectomy, bladder neck stricture, lifethreatening bleeding
Volume 3 Issue 5 - 2016

\author{
Mahmoudreza Moradi, Reza Aghelnezhad, \\ Hossein Abdi, Kaveh Kaseb, Haress Rezaee \\ Department of Urology, Tissue Engineering \& Regenerative \\ Medicine (TERM) research center of Kermanshah University of \\ Medical Sciences, Iran dr
}

Correspondence: Mahmoudreza Moradi, Urology Department, Tissue Engineering \& Regenerative Medicine (TERM) research center of Kermanshah University of Medical Sciences, Imam Reza Hospital, Iran, Tel 9891813248।I, Emailmrmoradi@gmail.com

Received: February 10, 2016 | Published: October 18, 2016

\section{Introduction}

Prostatectomy is one of the most common urologic procedures. Although TURP is now considered the standard procedure, open prostatectomy still plays a major role in urology. Simple prostatectomy should be considered when the obstructive tissue is estimated to weigh more than $75 \mathrm{~g}$, bladder diverticula, large bladder calculi, ankylosis of the hip or other orthopedic conditions that prevent proper positioning for TURP, recurrent or complex urethral conditions such as urethral stricture or previous hypospadias repair are other indications. ${ }^{1}$ However, blood loss is now minimal during prostatectomy and the need for a blood transfusion is uncommon but excessive hemorrhage had been a major concern ${ }^{1}$ and during enucleating of prostatic adenoma, profuse bleeding can occur ${ }^{2}$ by having all of these in view the 2003 American Urological Association guideline on the management of $\mathrm{BPH}$ reports a blood transfusion rate as high as $27 \%{ }^{3}{ }^{3}$ The most difficult situation one might face is profuse bleeding from the prostatic fossa with no obvious bleeding site. This usually happens after removal of very large adenomas and in patients with anticoagulation therapy or those who receives NSAIDs. In our experience this kind of bleeding did not respond to simple suture ligature or electrocautery.

With this scenario there are two recommendations available in the urologic literature. ${ }^{1}$ In Malament technique a No. 2 nylon pursestring suture can be placed around the bladder neck, brought out through the skin, and tied firmly as described by Malament. This maneuver closes the bladder neck and tamponades the prostatic fossa. The nylon suture is removed by cutting it at the skin and applying gentle traction on postoperative day 2 or 3 . Plicating sutures can be placed transversely in the posterior prostatic capsule to prevent further bleeding, as described by O'Coner. Both of the techniques are quite time consuming and difficult in a hemorrhagic field deep in the pelvic. The possibility of rectum trauma or tearing of the capsule while placing O'Coner plication sutures are two fearful concerns. Trauma to ureteral orifices, difficulties during suture removal and bladder neck stricture are Malament procedure complications. So it seems we need to a new technique so that can immediately and with low complication control life-threatening hemorrhage. Here we try to explain our experience with balloon tamponade of prostatic fossa in cases of persistent life-threatening bleeding after routine ligature of the arteries of the prostate which is required to control of bleeding.

\section{Materials and methods}

We retrospectively reviewed the 11 patients whom underwent balloon tamponade due to massive bleeding from prostatic fossa during open prostatectomy in our center between January 2007 and February 2016. We reviewed the patients' follow up visits with the mean of 42.09 months and also called the patients or their relatives to inquiry about their recovery and everything that might has happened to them after surgery, regarding their urologic status.

\section{Surgical technique}

In classic open prostatectomy, after enucleating of the adenoma, the prostatic fossa is inspected for residual tissue. If residual tissue found, was removed by sharp or blunt dissection. The prostatic fossa also must be examined for discrete bleeding sites that frequently can be controlled with an electrocautery or 4-0 chromic suture ligatures. In addition, a 0 -chromic suture is used to place two figure-of-eight 
sutures to advance the bladder mucosa into the prostatic fossa at the 5-o'clock and 7-o'clock positions at the prostatovesical junction to ensure control of the main arterial blood supply to the prostate. Fifty milliliters of water is then injected in the balloon of inserted $24 \mathrm{Fr}$ foley catheter to ensure that catheter balloon remains in the bladder does not retract into the prostatic fossa.

In our cases hemorrhage remained persistent despite the haemostatic Sutures, routine procedures for hemorrhage control and patients impeding to hypovolemic shock and required to massive transfusion protocol thus in transvesical prostatectomy cases $24 \mathrm{Fr}$ Foley catheter balloon inflated up to 80 or $100 \mathrm{~mL}$ in prostatic fossa and in retropubic prostatectomy cases after closure of prostate capsule we did same (Figure 1). In all cases a suprapubic Malecot catheter was used as cystostomy to evacuate irrigation fluid entered the bladder via foley catheter lodged in the prostatic fossa. On the first or second postoperative day the balloon fluid was emptied gradually with no case of recurrent bleeding. The foley might then become repositioned out of the fossa into the bladder without any difficulty. The foley removed on the second or third post-operative day if urine was clear.

\section{Results}

Overall 11 patients were studied with balloon in situ in prostatic fossa during open prostatectomy (Table1). The procedures were performed via suprapubic transvesical approach in 9 and retropubic in 2 patients. The mean patient's age was 74.81 years (range: 64-92). The indications for open prostatectomy were urinary retention in 7 patients, bladder stone in 2 and upper tract damage in 2 patients.

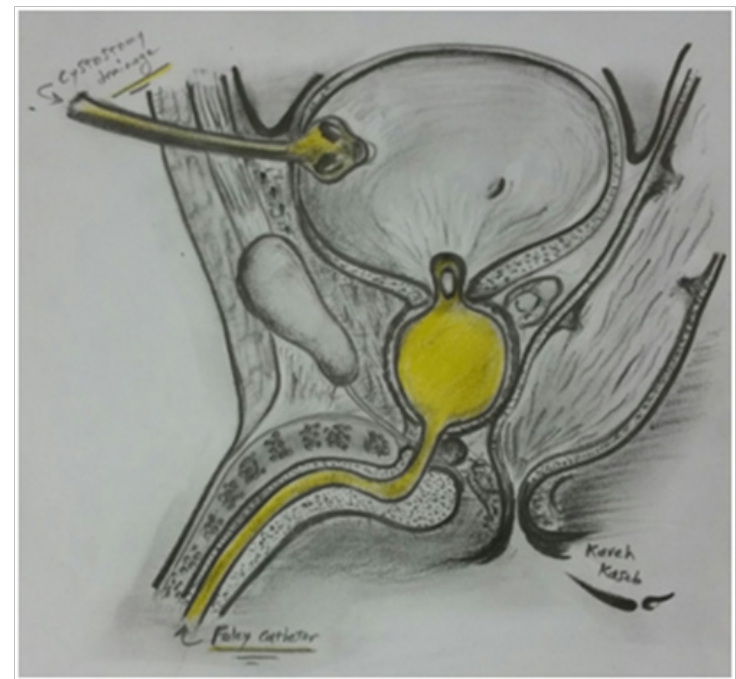

Figure 1 Inflated balloon of catheter in prostatic fossa.

Open prostatectomy was done in all patients who had large adenomas according to pre and intra operative observations. None of our patients had prostatic adenocarcinoma on their pathology. Fortunately, we didn't find any complications attributable to our technique. There was no bladder neck stricture, fistula formation or rectal injury even in those patients who underwent retropubic procedure. Intra operative transfusion was done in all patients but only 4 cases need to post operation blood transfusion.

Table I Patients characteristic and result of operations

\begin{tabular}{|c|c|c|c|c|c|c|c|c|c|c|}
\hline $\begin{array}{l}\text { Case } \\
\text { Number }\end{array}$ & Age(year) & $\begin{array}{l}\text { Indication } \\
\text { For } \\
\text { surgery }\end{array}$ & $\begin{array}{l}\text { Anticoagulant } \\
\text { use }\end{array}$ & $\begin{array}{l}\text { Length } \\
\text { of } \\
\text { Follow- } \\
\text { up } \\
\text { (month) }\end{array}$ & $\begin{array}{l}\text { Estimated } \\
\text { Intra. op } \\
\text { Blood } \\
\text { loss(ml) }\end{array}$ & $\begin{array}{l}\text { Surgical } \\
\text { Technique }\end{array}$ & $\begin{array}{l}\text { Intra op. } \\
\text { Transfusion }\end{array}$ & $\begin{array}{l}\text { Post op. } \\
\text { Transfusion }\end{array}$ & $\begin{array}{l}\text { Pre op. } \\
\text { Prostate } \\
\text { Volume }\end{array}$ & $\begin{array}{l}\text { Late } \\
\text { Complication }\end{array}$ \\
\hline I & 73 & AUR* & NO & 19 & 2500 & Sup. *** & 5 & 1 & 150 & no \\
\hline 2 & 64 & AUR & NO & 78 & 1200 & Sup. & 2 & 0 & $>100$ & no \\
\hline 3 & 69 & AUR & YES & 69 & 1300 & Retro***. & 2 & 0 & 85 & no \\
\hline 4 & 81 & Stone & $\mathrm{NO}$ & 64 & 1200 & Sup. & 2 & 1 & 90 & no \\
\hline 5 & 76 & $\uparrow \mathrm{Cr}$ & NO & 60 & 1300 & Sup. & 2 & 0 & 95 & no \\
\hline 7 & 72 & Stone & YES & 47 & 1000 & Sup. & 2 & 0 & 80 & no \\
\hline 8 & 69 & AUR & NO & 32 & 1000 & Sup. & 2 & I & 90 & no \\
\hline 9 & 92 & $\uparrow \mathrm{Cr}$ & NO & 29 & 1200 & Sup. & 2 & 0 & 100 & no \\
\hline 10 & 73 & AUR & $\mathrm{NO}$ & 10 & 1500 & Retro. & 3 & I & 120 & no \\
\hline 11 & 87 & AUR & $\mathrm{NO}$ & 4 & 1100 & Sup. & 3 & 0 & $>100$ & no \\
\hline
\end{tabular}

*Acute urinary retention $* *$ suprapubic $* * *$ retropubic 


\section{Discussion}

Till 20-25 years ago, open surgery was the most common approach to the prostate in benign prostatic hyperplasia. From the late 1970s, however, the development of endoscopes led to a gradual reduction in the number of open surgical operations for the prostate, and open prostatectomy continued to decrease rapidly in favor of the minimally invasive endoscopic techniques. Although TURP is considered the gold standard in treatment of $\mathrm{BPH}$, open prostatectomy is still a common practice with acceptable morbidity and very low mortality in most developing parts of world with no facilities for TURP and in developed countries for certain indications. ${ }^{4}$ The overall complication rate for open prostatectomy in one recent study ${ }^{5}$ was $40.1 \%(145 / 362)$ and the most common intraoperative complication in this procedure was bleeding. In a study of 902 patients $^{6}$ the overall complication rate was $17.3 \%$. The most relevant complications were bleeding requiring transfusion in 68 cases $(7.5 \%)$, urinary tract infection in $46(5.1 \%)$ and surgical revision due to severe bleeding in 33 (3.7\%).

Recent surgical techniques have had a significant effect on intraoperative bleeding and made it a less fearful scenario ${ }^{1}$ but it is still a concern and in cases who bleeding are occurred one must be able to deal with the situation as fast and effective as possible to reduce morbidity and prevent mortality in this usually senile group of patients with co-morbidities. According to previous studies reducing operation time and blood loss is more beneficial in comparison to decreasing morbidity and mortality. On the other hand blood transfusion by itself can cause many complications ${ }^{7}$ which mandates avoiding transfusion with proper use of haemostatic maneuvers. The most difficult situation one might face is profuse bleeding from the prostatic fossa with no obvious bleeding site. This usually happens after removal of very large adenomas and in patients with anticoagulation therapy or those who receives NSAIDs. Unfortunately, these are the same as the most sensitive groups to hemorrhagic complication and the most susceptible patients to severe morbidities and mortality. In our present experience this kind of bleeding in all patients did not respond to simple suture ligature or electrocautery.

Also there are different techniques in order to post simple prostatectomy bleeding control as O' Coner and Malament which have serious complications and the last way to control of this scenario is most aggressive procedure- internal iliac artery ligation so the need to new technique with minimal complication to prevent of such condition is really undeniable. Nakahara-ku et al. ${ }^{2}$ advised the bilateral internal iliac arteries are transient occlusion with Bulldog clamps if volume of $\mathrm{BPH}$ is more than $100 \mathrm{~mL}$ but it is not always applicable. Our new technique is much easier and faster for cessation of profuse life threatening hemorrhages and termination of the operation as soon as possible to lessen the patient's stress and morbidity. It's enough to adjust the balloon of a $24 \mathrm{Fr}$ foley in the fossa and gently inflating it to fill the fossa. There is no need to over inflate the balloon in order to apply pressure to the capsule so there is no concern regarding rupture. With the technique described here, bleeding usually stops completely under the surgeon's direct vision. There might be a smaller degree of bloodshed trough the urethra which can be efficiently managed by wrapping gauze around the folly just where it leaves the meatus. This occludes the urethra and helps the clot to form around foley catheter.

We preferred to deflate the balloon as soon as possible and reposition the foley within bladder instead of the fossa. It is safe to do this on the first or second post operative days if the irrigation fluid is free of clots and in the absence of significant hemorrhage. The foley itself could be removed later on the second or third day after surgery. According to our knowledge there is not any previous report regarding this technique in the literature. Hooman djalalat et al. ${ }^{8}$ reported the use of a novel catheter which its balloon places inside the prostatic fossa but their intention was to exclude urethral catheter and they used this in all patients regardless of degree of bleeding. They did not mentioned anything regarding bleeding in their article but they didn't find any harm attributable to inflation of the balloon in the prostatic fossa. We didn't find any complication as well. None of our patients had bladder neck stricture. The technique which we present here is a very simple and rapid one, but it is able to save patients' lives and reduces morbidities related to severe bleeding and massive transfusion. Although it's not wise to use the technique mentioned above as a routine practice; one must consider its use in case of severe bleeding from prostatic fossa while or soon after open prostatectomy. It has no effect on bleeding from a distinct vessel ending specially those located on 5 and 7 o'clock positions in the bladder neck.

\section{Conclusion}

Balloon tamponade is a simple, quick and lifesaving new technique which is used when persistent and significant bleeding from the prostatic fossa continues after simple prostatectomy. This technique in our opinion is superior to the other previously introduced techniques, but of course must be reserved for exceptional life-threatening situations until further reports verify its safety.

\section{Acknowledgments}

None.

\section{Conflicts of interest}

The author declares there is no conflict of interest.

\section{References}

1. Alan J wein, Louis R Kavoussi, Alan W Patrin. CAMPBELL-WALSH UROLOGY. Chapter 106, 2016. p. 2535-2545.

2. Takeuchi T, Zaitsu M, Mikami K, et al. Transient occlusion of bilateral internal iliac arteries facilitates bloodless operative field in subcapsular prostatectomy. Case Rep Med. 2012;2012:812615.

1. AUA Practice Guidelines Committee. AUA Guideline on Management of Benign Prostatic Hyperplasia. Chapter 1: diagnosis and treatment recommendations. J Urol. 2003;170(2):e530-e547.

2. Ahmed Gadam I, Nuhu A, Aliyu S. Ten-year experience with open prostatectomy in maiduguri. ISRN Urol. 2016;2012:406872.

3. Oranusi CK, Nwofor A, Oranusi IO. Complication rates of open transvesical prostatectomy according to the Clavien-Dindo classification system. Niger J Clin Pract. 2012;15(1):34-37.

4. Gratzke C, Schlenker B, Seitz M, et al. Complications and early postoperative outcome after open prostatectomy in patients with benign prostatic enlargement: results of a prospective multicenter study. $J$ Urol. 2007;177(4):1419-1422

5. Shirazi M, Ghaffari S, Hassanpour A, et al. Urethral Catheter Traction Reduces Bleeding Compared With Suturing of Prostatic Vesical Junction During Suprapubic Prostatectomy: A Randomized Clinical Trial Study. Urology. 2009;74(1):137-141.

6. Djaladat H, Mehrsai A, Saraji A, et al. Suprapubic Prostatectomy With a Novel Catheter. J Urol. 2006;175(6):2083-2086. 